\title{
Estudio comparativo de dos tipos de agujas en hemodiafiltración en línea de alta eficacia
}

\author{
Ana Vanessa Fernández Martínez, Verena Romero Willholft, Laura Pérez Valencia, Alicia Moreno Vallejo, \\ Tania Ortega Gómez, Salvadora Soto Ureña
}

Enfermeras centros de hemodiálisis Fresenius Medical Care Services Cartagena y San Pedro del Pinatar. Murcia

\section{Resumen}

La hemodiafiltración on line post dilucional es la técnica más eficaz en la depuración de moléculas de diferentes pesos.

El volumen convectivo y la dosis de diálisis pueden estar relacionado con la supervivencia del paciente.

Ambos, parámetros están influenciados por el flujo sanguíneo, habiendo sido debatido el uso de diferentes calibres de aguja en lo referente a resultados de eficiencia y valoración de dolor.

\section{Objetivo:}

Comparar la eficacia, seguridad, comodidad y sensación de dolor entre el catéter fístula y las agujas convencionales en el paciente en hemodiafiltración en línea de alta eficacia.

Estudio prospectivo cruzado sobre población prevalente en hemodiafiltración en línea posdilucional, Se analizan variables demográficas, hemodinámicas del acceso vascular, de seguridad y escala de dolor y comodidad pre-post para el enfermero en $\mathbf{1 5 8 4}$ sesiones.

Analisis estadistico SPSS 13.0. Significación $p<$ 0,05 .

\author{
Correspondencia: \\ Ana Vanessa Fernández Martínez \\ Centro de diálisis de Cartagena \\ FMC Services Murcia \\ Paseo Alfonso XIII, 61 \\ 30203 Cartagena. Murcia \\ E-mail:nefroclubcarthago@gmail.com
}

No diferencias en Presion arterial, Presion venosa y recirculación. Sí en flujo sanguineo, siendo superior con las supercath.

En eficacia, diferencias significativas en $\mathrm{Kt}$ $(p=0,04)$, VTR $(p=0,00)$ y litros de sangre dializada $(p=0,01)$, supercath $(63,1),(27,4),(113,1)$ versus convencional $(60,9),(25,3),(108,5)$ respectivamente.

Valoración enfermera de comodidad significativamente $(p=0,00)$ mejor con las agujas convencionales tanto en conexión como desconexión. Percepción de dolor para el paciente es mayor con supercath.

\section{PALABRAS CLAVE:}

- EFICACIA DIALÍTICA

- DOLOR Y VALORACIÓN ENFERMERA

Comparative study of two types of needles in high-efficiency online haemodiafiltration

\section{Abstract}

Post-dilution online haemodiafiltration is the most efficacious in removing molecules of different weights.

The convective volume and the dialysis dose may be related to patient survival.

Both parameters are influenced by blood flow, and the use of different needle calibres has been debated with regard to results in efficiency and pain assessment. 


\section{Objective:}

To compare the efficacy, safety, convenience and pain perception between fistula catheter and conventional needles in patients undergoing high-efficiency online haemodiafiltration.

Prospective cross-sectional study of prevalent population on post-dilution online haemodiafiltration. The variables analysed were demographic, vascular access haemodynamic, safety, pain scale and prepost convenience for the nurse, over 1584 sessions.

Statistical analysis SPSS 13.0. Significance $p<$ 0.05 .

No differences in arterial pressure, venous pressure and recirculation. Differences were found in blood flow, which was higher with supercath.

In efficiency, there were significant differences in Kt $(p=0.04)$, VTR $(p=0.00)$ and litres of dialysed blood ( $p=0.01)$, supercath (63.1), (27.4), (113.1) compared to conventional (60.9), (25.3), (108.5) respectively.

The nursing assessment of convenience was significantly $(p=0.00)$ better with conventional needles, both in connection and disconnection. Pain perception in patients is higher with supercath.

\section{KEY WORDS:}

- DIALYSIS EFFICACY

- PAIN AND NURSING ASSESSMENT

\section{Introducción}

La hemodiafiltracion on-line de alta eficacia puede reducir la mortalidad del paciente en hemodiálisis ${ }^{1-3}$. Los recientes resultados del estudio $\mathrm{ESHOL}^{1}$ muestran una reducción del $30 \%$ en la mortalidad global, $33 \%$ en la mortalidad cardiovascular y $55 \%$ en la de origen infeccioso. Estos datos son congruentes con los subanalisis del estudio CONTRAST ${ }^{2}$ y TURKEY $^{3}$, en los que encuentran esa reducción de mortalidad en los tertiles de mayor volumen convectivo. Así pues, el volumen convectivo puede estar relacionado con la supervivencia del paciente, aunque diferentes aspectos metodológicos, como un flujo sanguíneo inadecuado o un tiempo de diálisis inferior a 240 minutos por sesión, pueden impedir alcanzar los objetivos convectivos previstos en la HDFOL, y por tanto, limitar su eficacia ${ }^{4}$.
Por otro lado, la dosis de diálisis es considerada fundamental en la diálisis adecuada y en la supervivencia del paciente ${ }^{5}$. La medición de dicha dosis mediante el $\mathrm{Kt}$ ha sido considerada por diferentes autores como un método mas exigente que mediante el $\mathrm{Kt} / \mathrm{V}$, ya que este ultimo puede infraestimar los casos de diálisis inadecuada ${ }^{6-7}$.

Ambos, Kt y volumen de reinfusión (VTR) están influenciados por el flujo sanguíneo ${ }^{8}$, habiendo sido debatido el uso de diferentes calibres de aguja en lo referente a resultados de eficiencia y valoración de dolor.

El catéter fístula supercath, una nueva cánula de punción de fluoruroplástico, presenta una menor calibre de punción con un mayor calibre interno, permitiendo alcanzar mayores flujos sanguíneos con menor daño en la pared del acceso $0^{9-10}$.

El objetivo del presente estudio fue comparar la eficacia, seguridad, comodidad del enfermero y sensación de dolor del paciente entre el catéter fístula y las agujas convencionales en el paciente en HDFOL posdilucional.

\section{Pacientes y métodos}

Estudio prospectivo doble cruzado sobre población prevalente en HDFOL posdilucional de alta eficacia, con fístula arteriovenosa autóloga (FAVI) en territorio braquial o protésica (PTFE). La duración del estudio fue de 16 semanas, 48 sesiones por paciente. Se reclutan 33 pacientes. 17 inician durante 12 sesiones (FI) con catéter Supercath y el resto con agujas convencionales de 14 o 15G. Se cruzan a las 12 sesiones (F2). Posteriormente 2 fases mas de 12 sesiones de duración cada una ( $F 3=F 1$ y F2 $=F 4$ ). Todas las sesiones con monitor ST 5008, reinfusión automática del volumen convectivo, y autoflujo de Qd factor 1 . Qb máximo con limite de 500 $\mathrm{ml} / \mathrm{min}$, calculado en función de presiones arteriales $(P A)$ y venosas (PV) (máximo $\pm 250 \mathrm{mmHg}$ ), y recirculación (REC) ( $\leq 20 \%)$.. El esquema terapéutico de cada sesión es igual en las 4 fases.

Se analizan las siguientes variables en estudio: demográficas (sexo, edad, causa, permanencia), hemodinámicas del acceso vascular (flujo sanguíneo real (Qb), PA, $\mathrm{PV}, \mathrm{REC}$ ), de eficacia ( $\mathrm{K}$, litros de sangre depurada, VTR), de seguridad (extravasaciones, punciones repetidas, tiempo de hemostasia entre 5 y 10 minutos o mayor, estado de cámaras y dializador después de la sesión) de 
comodidad (escala de 1 a 5 de valoración subjetiva del enfremero/a pre y postdiálisis), y de dolor (escala 1 a 5 de valoración subjetiva del paciente postpunción).

Para el análisis estadístico se utiliza el paquete SPSS 13.0 para Windows. Las variables cuantitativas se expresan como media y desviación estándar. Para el contraste de hipótesis se utiliza la $t$ de Student para muestras relacionadas (valor promedio $\mathrm{Fl}-\mathrm{F} 3$ versus F2-F4). Las variables cualitativas se expresan como frecuencias y porcentajes, utilizando para su contraste la chi cuadrado de Pearson. Se establece significación si $p<0,05$.

\section{Resultados}

Finalizan el estudio 33 pacientes, de los cuales un $60,4 \%$ hombres, con una edad media de 67,6 , el $94 \%$ portadores de FAVI. El calibre de aguja utilizado es en un $76 \% 15 \mathrm{G}$ y $24 \% 14 \mathrm{G}$. Se analizan un total de 1584 sesiones.

En cuanto a los parámetros hemodinámicos, el Qb promedio obtenido con el catéter Supercath es significativamente mayor $(p=0,02)$ que el alcanzado con las agujas convencionales $(463,95 \pm 29,51 \mathrm{ml} / \mathrm{min}$ versus $444,68 \pm 29,03 \mathrm{ml} / \mathrm{min}$ ). No se encuentran diferencias en PA, PV y REC (tabla 1 ).

\begin{tabular}{|c|c|c|c|}
\hline Parámetros & Catéter Supercath & $\begin{array}{c}\text { Aguja conven- } \\
\text { cional }\end{array}$ & $P$ \\
\hline $\mathrm{PA}, \mathrm{mmHG}$ & $-201,91 \pm 30,35$ & $-213,84 \pm 25,57$ & 0,062 \\
\hline $\mathrm{PV}, \mathrm{mmHg}$ & $211,18 \pm 23,81$ & $206,59 \pm 22,11$ & 0,440 \\
\hline REC, \% & $18,64 \pm 4,70$ & $18,59 \pm 4,35$ & 0,970 \\
\hline
\end{tabular}

Tabla 1. Valores de presión arterial, presión venosa y recirculación.

En cuanto a variables de eficacia, encontramos valores significativamente mayores con el catéter Supercath en $\mathrm{Kt}(p=0,04)$, VTR $(p=0,008)$ y en litros de sangre dializada $(p=0,01)$, tal y como se muestra en el gráfico 1 .

No encontramos diferencias significativas en cuanto al número de extravasaciones en ambos periodos de estudio, aunque se repiten más punciones con Supercath ( $10,4 \%$ versus $3,5 \%, p<0,001)$. El $48,9 \%$ de las sesiones con Supercath presentan un tiempo de hemostasia entre 5 y 10 minutos, frente al $40,4 \%$ con convencional $(p=0,018)$. No hay diferencias en el estado final de suciedad/coágulos ni en cámaras del circuito ni dializador.

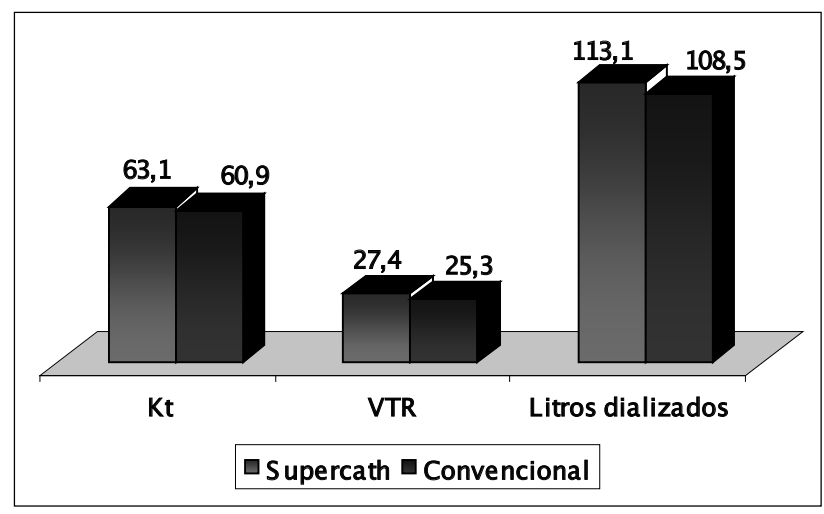

Gráfico 1. Parámetros de eficacia.

La valoración enfermera de comodidad es significativamente $(p<0,001)$ mejor con las agujas convencionales tanto en conexión como en desconexión. La percepción de dolor para el paciente es mayor con Supercath $(p<0,001)$. Los resultados se muestran en el gráfico 2.

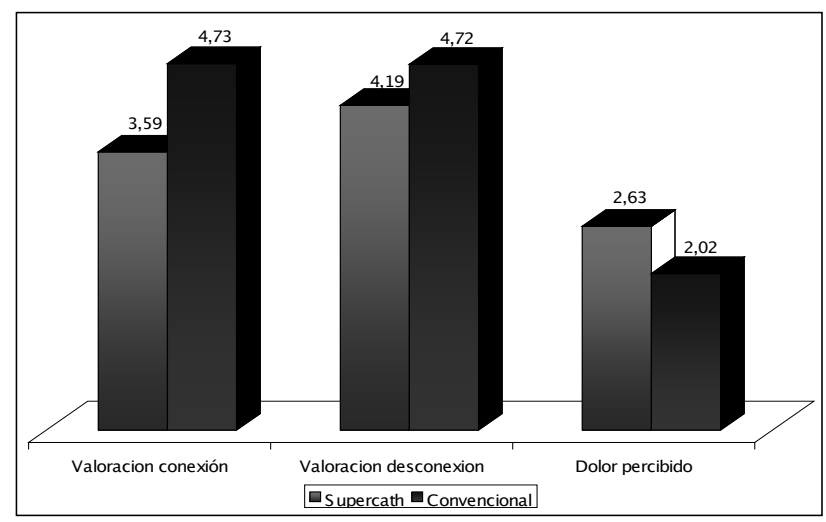

Gráfico 2. Valoración enfermera y dolor percibido por el paciente.

\section{Discusión}

Los resultados de nuestro estudio son congruentes con los manifestados por otros estudios en cuanto al aumento de flujos sanguíneos al incrementar el calibre de la aguja, disminuyendo o manteniendo las mismas presiones arteriales y venosas si trabajamos a mismo flujo de bomba ${ }^{9}$ o como en nuestro caso estableciendo un límite superior de $250 \mathrm{~mm} / \mathrm{Hg}^{8}$.

Las características especiales del catéter Supercath hacen que el calibre interno del catéter (equivalente a 14G) sea superior al externo de la aguja de punción (16G). Por ello, en nuestro estudio se constata una mayor eficacia con respecto a las agujas convencionales, tal como estaba previamente descrito ${ }^{9}$. Sin embargo, nuestros resultados muestran un incremento en el flujo sanguíneo real ( $20 \mathrm{ml} / \mathrm{min})$, $\mathrm{Kt}$ (2,3 litros) y volumen de sangre dializada ( 4,6 litros) sensiblemente mayores que 
en lo reportado por Martínez Ocaña y cols $(9 \mathrm{ml} / \mathrm{min}$, 1,83 litros y 2,3 litros, respectivamente). Las diferencias metodológicas $(\mathrm{Qb}$ obtenido por presiones en nuestro caso y fijados por bomba en el suyo) así como las diferencias de tamaño muestral justifican esta disparidad.

Del mismo modo, resulta muy destacable el incremento del VTR de más de 2 litros en nuestros resultados, pese a partir de valores promedio superiores a 25 litros con agujas convencionales. De esta manera, el uso del catéter Supercath se convierte en una alternativa de mejora en la HDFOL posdilucional de alta eficacia. Este hecho no es nada desdeñable, habida cuenta que los problemas metodológicos en otros estudios ${ }^{2-3}$, con especial importancia del Qb, hicieron que solo una tercera parte de los pacientes alcanzaran el objetivo convectivo deseado, y justo en esos tertiles es donde se aprecio un beneficio en la supervivencia ${ }^{4}$.

Los mejores resultados en el tiempo de hemostasia del catéter Supercath en nuestro estudio, pueden ser debidos al menor trauma en la punción. Estos datos no fueron obtenidos por Martínez Ocaña y cols, quizás por utilizar una medida diferente (en nuestro caso por intervalos y en el suyo por minutos).

Por otro lado, la percepción del paciente de un incremento en la percepción del dolor es congruente con lo descrito por otros 9 , aunque en ambos estudios la limitación es la subjetividad en la medida, ya que no existen instrumentos específicos de medida en el paciente en hemodiálisis ${ }^{10}$. En cualquier caso, la puntuación promedio obtenida $(2,63)$ se encuentra en la mitad de la escala, lo cual también puede verse influido por la probable inseguridad transmitida por el personal de enfermería, no acostumbrado previamente al manejo de este tipo de catéter. De hecho, los peores resultados de valoración enfermera y de dolor percibido corresponden a las fases 1 y 2 , aunque el diseño del estudio no permite de forma objetiva aclarar las razones de una posterior mejora.

\section{Conclusiones}

El uso del catéter fístula Supercath nos permite conseguir mayor flujo sanguíneo, sin incremento de presiones tanto arteriales como venosas ni recirculación, lo que nos hace conseguir mayor eficacia dialítica y un mayor volumen convectivo.

Si bien, la valoración tanto del enfermero como del paciente en escala de seguridad, comodidad y dolor es peor con Supercath, estos resultados se producen fundamentalmente en las fases 1 y 2 , por lo que una vez más se requiere del conocimiento y perfeccionamiento de la técnica del enfermero nefrológico.

\section{Agradecimientos}

Agradecer al Servicio de Nefrología del Hospital Santa Lucía de Cartagena y a Izasa, por su contribución para el desarrollo de este trabajo.

Recibido: 8 Agosto 2013
Revisado: 16 Agosto 2013
Modificado: 25 Agosto 2013
Aceptado: 26 Agosto 2013

\section{Bibliografía}

1. Maduell F, Moreso F, Pons M, Ramos R, Mora-Macià $J$, Carreras $J$ et al. High-Efficiency Postdilution Online Hemodiafiltration. Reduces All-Cause Mortality in Hemodialysis Patients. Journal of the American Society of Nephrology. 2013; 24: 1-11.

2. Muriel P.C.Grooteman, Marius A, Michiel L Bots, E. Lars Penne, Neelke C. van der Weerd, Albert H.A. Mazairac et al. Effect of Online Hemodiafiltration on All-Cause Moortality and Cardiovascular Outcomes. 2012; J AmSoc Nephrol 23: 1087-1096.

3. Ercan Ok, Gulay Asci, Ebru Sevinc Ok, Fatih Kircelli, Mumtaz Yilmaz, Ender Hur et al; On behalf of the 'Turkish Online. Haemodiafiltration Study': Mortality and cardiovascular events in online haemodiafiltration (OL-HDF) compared with highflux dialysis: Results from the Turkish OL-HDF Study. Nephrol Dial Transplant 28:192-202, 2013.

4. Canaud B, Bourie SK. Emerging clinical evidence on online hemodiafiltration: does volume of ultrafiltration matter? Blood Purif 35: 55-62, 2013.

5. Chertow GM, Owen WF, Lazarus JM y cols. Exploring the reverse $\mathrm{j}$-saped curve between urea production ratio and mortality. Kidney Int 1999; 56: 1872-1878. 
6. Fernández AV, Soto $S$, Arenas $M$ y cols. Estudio comparativo de la dosis de dialisis medida por (Kt) y KtV. 21 Rev Soc Esp Enferm Nefrol 2009; 12(2): 97-102.

7. Maduell F, Vera M, Serra N y cols. Kt como control y seguimiento de la dosis en una unidad de hemodiálisis. Nefrología 2008; 28: 43-47.

8. Fernández $A V$, Caparros $V$, Horrillo $F$ y cols. La eficacia de la hemodiafiltración online es de pendiente del flujo sanguíneo. Entonces, ¿Por qué no incrementamos el tamaño de las agujas?. En: Libro de comunicaciones presentadas al XXXVI
Congreso Nacional de la SEDEN. Hospal/SEDEN. Sevilla 2011.

9. Martínez Ocaña 0, Rodríguez Estaire J, Ruiz Sanz B, Martínez Navarro JA, Merida Herrero E. Catéter- fistula: una nueva alternativa en la punción de accesos vasculares. Rev Soc Esp Enferm Nefrol. 2010; 13 (2): 105 -111.

10. Rodríguez MA, Hernández D, Juan F, Calls J. Evaluación del dolor crónico en una población de pacientes hemodializados. Rev Soc Esp Enferm Nefrol 2007; 10 (2): 137-143. 